TRANSACTIONS OF THE

AMERICAN MATHEMATICAL SOCIETY

Volume 361, Number 1, January 2009, Pages 207-222

S 0002-9947(08)04650-3

Article electronically published on July 30, 2008

\title{
HOMOTOPY GRAPH-COMPLEX FOR CONFIGURATION AND KNOT SPACES
}

\author{
PASCAL LAMBRECHTS AND VICTOR TURCHIN
}

\begin{abstract}
We prove that the primitive part of the Sinha homology spectral sequence $E^{2}$-term for the space of long knots is rationally isomorphic to the homotopy $\mathcal{E}^{2}$-term. We also define natural graph-complexes computing the rational homotopy of configuration and of knot spaces.
\end{abstract}

\section{INTRODUCTION}

In [28, 29] D. Sinha defined a cosimplicial model for the space $E m b$ of long knots $\mathbb{R} \hookrightarrow \mathbb{R}^{d}, d \geq 4$. It was proven in [21] that the associated homology Bousfield-Kan spectral sequence collapses rationally at the second term 1 The same result was established for the associated homotopy spectral sequence (over $\mathbb{Q}$ ). The proof will appear in 2. But $E m b$ is an $H$-space with a homotopy commutative product 2 It implies in particular that the $\mathcal{E}^{2}$-term of the (co)homotopy spectral sequence must be rationally isomorphic to the primitive part of the $E^{2}$ (co)homology term.
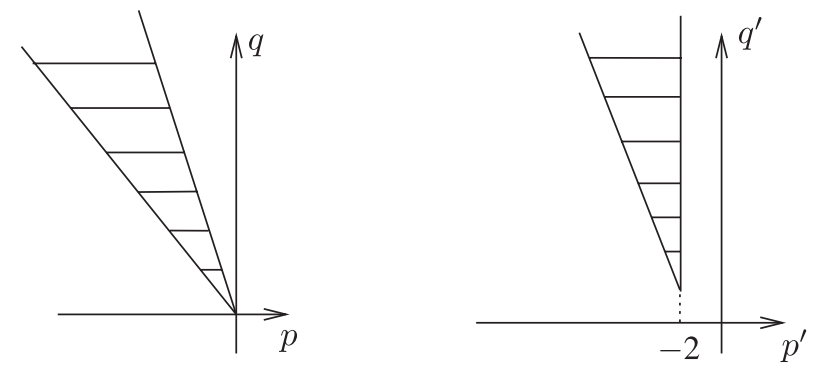

Figure 1. Homology $E_{p, q}^{2}$ and homotopy $\mathcal{E}_{p^{\prime}, q^{\prime}}^{2}$ terms.

Received by the editors November 27, 2006.

2000 Mathematics Subject Classification. Primary 57Q45; Secondary 55P62, 57R40.

Key words and phrases. Knot spaces, embedding calculus, Bousfield-Kan spectral sequence, graph-complexes.

The first author is chercheur qualifié au F.N.R.S.

The second author was supported in part by the grants NSH-1972.2003.01 and RFBR 05-0101012a.

${ }^{1} \mathrm{~A}$ similar result was obtained in 3 .

${ }^{2}$ It was shown recently by P. Salvatore that $E m b$ is a double loop space [26].

(C)2008 American Mathematical Society Reverts to public domain 28 years from publication 
The (co)homology $E_{p, q}^{2}$-term is concentrated in the second quadrant between two lines [32]:

$$
\begin{array}{ll}
q=-\frac{d-1}{2} p & \text { (lower line) } \\
q=-(d-1)(p+1) & \text { (upper line). }
\end{array}
$$

The (co)homotopy $\mathcal{E}_{p^{\prime}, q^{\prime}}^{2}$-term is also concentrated in the second quadrant and bounded by the lines [27]:

$$
\begin{array}{ll}
q^{\prime}=-(d-2) p^{\prime}-d+3 & \text { (lower line) } \\
p^{\prime}=-2 & \text { (right line). }
\end{array}
$$

In Part 1 of the paper we will give a simple and purely algebraic proof of this isomorphism:

$$
\mathcal{E}_{*, *}^{2}=\operatorname{Prim}\left(E_{*, *}^{2}\right) .
$$

In particular we will see in Section 4 how via this isomorphism the bigradings of both spectral sequences are related to each other. We will see that the lower line of the homotopy spectral sequence corresponds to the lower line of the homology one. The right line $p^{\prime}=-2$ of $\mathcal{E}^{2}$ corresponds to the upper line $q=-(d-1)(p+1)$ of $E^{2}$. In general any vertical line $p^{\prime}=-n$ corresponds to $q=-(d-1)(p+n-1)$.

This isomorphism in the case of the lower lines (on the level of the bialgebra of chord diagrams) was proved by J. Conant [9]. He gives an elegant reformulation of his result using 3-valent graphs.

Part 2, which actually gave the name to the paper, is devoted to graph-complexes. Our motivation was to produce new graph-complexes whose homology has a nice geometrical interpretation. We define a series of graph-complexes that compute the rational homotopy of configuration spaces. Building upon this series of complexes we define a bigger complex whose homology is the rational homotopy of the space of long knots. A more thorough introduction for Part 2 is Section 7.

Part 1. Isomorphism $\mathcal{E}^{2}=\operatorname{Prim}\left(E^{2}\right)$

\section{Cosimplicial model For the SPACE OF LONG KNOTS MODULO IMMERSIONS}

The space $\overline{E m b}$ of long knots modulo immersions is the homotopy fiber of the inclusion

$$
E m b \hookrightarrow I m m
$$

of the space of long knots Emb in the space of long immersions $I m m$. By the word "long" we understand smooth maps $\mathbb{R}^{1} \rightarrow \mathbb{R}^{d}$ that coincide with a fixed linear map outside a compact subset of $\mathbb{R}^{1}$. (We will deliberately omit $d$ to simplify notation, assuming that the dimension $d \geq 4$ is fixed once and forever 3 )

D. Sinha showed in 29] that $\overline{E m b}$ is homotopy equivalent to $E m b \times \Omega I m m \simeq$ $E m b \times \Omega^{2} S^{d-1}$. So, the homology and homotopy of $E m b$ are easily related to those of $\overline{E m b}$ and the results for $\overline{E m b}$ that we obtain in the first part of the paper can be obviously reestablished for $E m b$.

In 28] D. Sinha defined a cosimplicial space whose homotopy totalization is $\overline{E m b}$. The $n$-th component $C^{n}$ of the cosimplicial space is some compactification

\footnotetext{
${ }^{3}$ When we consider configuration spaces we assume $d \geq 3$.
} 
of the configuration space of points in $I \times \mathbb{R}^{d-1}=[0,1] \times \mathbb{R}^{d-1}$ :

$$
\left\{\begin{array}{l|l}
\left(x_{0}, x_{1}, \ldots, x_{n+1}\right) & \begin{array}{ll}
x_{i} \in I \times \mathbb{R}^{d-1} ; & x_{i} \neq x_{j} \\
x_{0}=(0, \overline{0}) ; & x_{n+1}=(1, \overline{0})
\end{array}
\end{array}\right\} .
$$

Given $0 \leq i \leq n+1$, the coface map $d^{i}$ is the doubling of the $i$-th point $x_{i}$ of the configuration in the direction $(1, \overline{0})$. The codegeneracy map $s^{i}$, for $i=1, \ldots, n$, is given by the forgetting of $x_{i}$.

In 29] D. Sinha provides another "operadic" construction for the cosimplicial replacement of $\overline{E m b}$ (this cosimplicial space is homotopy equivalent to the previous one).

For any symmetric monoidal category $(\mathcal{C}, \otimes, \mathbf{1})$, with product $\otimes$ and unit $\mathbf{1}$, denote by $\mathcal{A S S}=\{\mathbf{1}\}_{n \geq 0}$ the associative non- $\Sigma$ operad 4

Provided an operad $\mathcal{O}$ in $(\mathcal{C}, \otimes, \mathbf{1})$ is endowed with a morphism $\mathcal{A S S} \rightarrow \mathcal{O}$, the collection $\left\{\mathcal{O}^{n}\right\}_{n \geq 0}=\{\mathcal{O}(n)\}_{n \geq 0}$ becomes a cosimplicial object in the category $\mathcal{C}$. Cofaces $d^{i}: \mathcal{O}^{n} \rightarrow \mathcal{O}^{n+1}, i=0, \ldots, n+1$, are compositions with $m=\mathbf{1}=\mathcal{A S S}(2)$ :
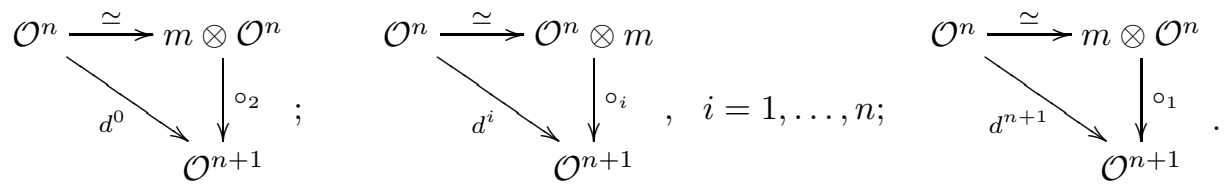

Codegeneracies are compositions with $e=\mathbf{1}=\mathcal{A S S}(0)$ :

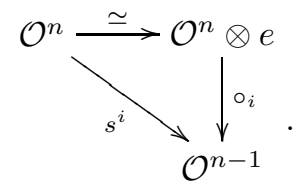

Sinha applies this standard construction to an operad $\{C\langle n\rangle\}_{n \geq 0}$ homotopy equivalent to the operad of little $d$-cubes. Each space $C^{n}=C\langle n\rangle, n \geq 2$, of this operad is the closure in $\left(S^{d-1}\right)^{\left(\begin{array}{c}n \\ 2\end{array}\right)}$ of the space of reciprocal directions of $n$ distinct points in $\mathbb{R}^{d}$ :

$$
\left\{\left(\frac{x_{j}-x_{i}}{\left|x_{j}-x_{i}\right|}\right)_{1 \leq i<j \leq n} \mid \begin{array}{c}
x_{i} \in \mathbb{R}^{d} ; \\
x_{i} \neq x_{j} \text { for all } i \neq j
\end{array}\right\} \subset\left(S^{d-1}\right)^{\left(\begin{array}{c}
n \\
2
\end{array}\right)} .
$$

He also defines $C\langle 0\rangle=C\langle 1\rangle=*$.

We will assume that we work with one of these cosimplicial models. The operadic and cosimplicial structures of $C^{\bullet}$ induce similar structures on the (co)homology and (co)homotopy of $C^{\bullet}$. The cohomology simplicial algebra will be denoted by

$$
A_{\bullet}=\left\{A_{n}\right\}_{n \geq 0}=\left\{H^{*}\left(C^{n}\right)\right\}_{n \geq 0} .
$$

We will consider the cohomology only with rational coefficients.

The rational cohomotopy simplicial Lie coalgebra will be denoted by

$$
L_{\bullet}=\left\{L_{n}\right\}_{n \geq 0}=\left\{\operatorname{Mor}\left(\pi_{*}\left(C^{n}\right), \mathbb{Q}\right)\right\}_{n \geq 0} .
$$

We will also work with the rational homotopy cosimplicial Lie algebra

$$
L^{\bullet}=\left\{L^{n}\right\}_{n \geq 0}=\left\{\pi_{*}\left(C^{n}\right) \otimes \mathbb{Q}\right\}_{n \geq 0}
$$

\footnotetext{
${ }^{4}$ Dually we will denote by $\mathcal{C O} \mathcal{A S S}=\{\mathbf{1}\}_{n \geq 0}$ the associative non- $\Sigma$ cooperad in $(\mathcal{C}, \otimes, \mathbf{1})$
} 


\section{Explicit Description of $A_{n}=H^{*}\left(C^{n}\right)$ AND of $L^{n}=\pi_{*}\left(C^{n}\right) \otimes \mathbb{Q}$}

The algebras $A_{n}, n \geq 0$, are well known [1, 17. Being graded commutative they are generated by $a_{i j}, 1 \leq i \neq j \leq n$, of degree $d-1$, that satisfy the relations:

$$
\begin{array}{ll} 
& a_{j i}=(-1)^{d} a_{i j}, \\
\text { quadratic } & a_{i j}^{2}=0, \\
\text { relations } & a_{i j} a_{j k}+a_{j k} a_{k i}+a_{k i} a_{i j}=0 .
\end{array}
$$

To recall, the component $C\langle 0\rangle$ is a point, so $A_{0}=\mathbb{Q}$.

Any monomial can be viewed as a directed graph on the set $\{1,2, \ldots, n\}$ : the directed edge $(i, j)$ appears exactly the number of times the generator $a_{i j}$ is represented in the monomial; see Figure 2.

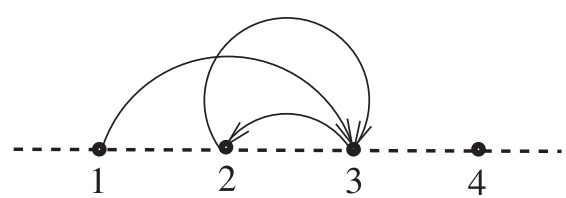

Figure 2. Graph corresponding to $a_{13} a_{32} a_{23} \in A_{4}$.

It can be easily seen that a monomial in $A_{n}$ is non-zero if and only if the corresponding graph is a forest. On such a graph $\Gamma$ the face map $d_{0}(\Gamma)$ is non-zero if and only if the valence of vertex 1 is zero. In this case $d_{0}$ simply removes the vertex 1 , all other vertices are shifted by 1 . The face $d_{i}(\Gamma), i=1, \ldots, n-1$, is obtained by collapsing the segment $[i, i+1]$ (the vertices $\geq i+1$ all being shifted by 1$)$. If $\Gamma$ contains the edge $(i, i+1)$, then $d_{i}(\Gamma)=0$. The face $d_{n}$ acts similarly to $d_{0}$, removing the last $n$-th vertex.

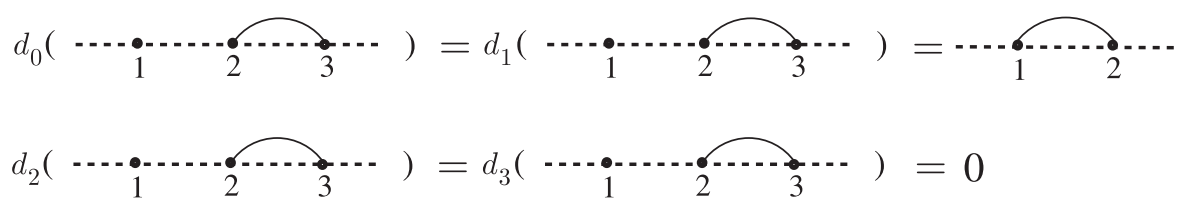

FigURE 3

The degeneracy map $s_{i}$, for $i=1, \ldots, n+1$, inserts a new vertex between $i-1$ and $i$.

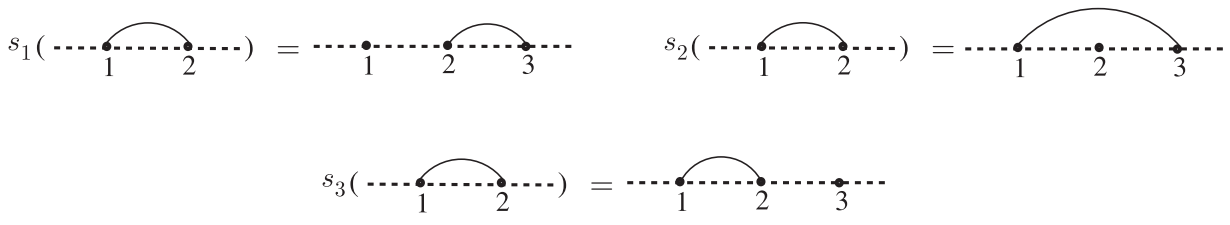

FigURE 4 
The normalized part $N A_{n}$ of $A_{n}$,

$$
N A_{n}=A_{n} /+_{i=1}^{n} \operatorname{Im} s^{i}
$$

is spanned by the forests with each vertex $1, \ldots, n$ of positive valence.

The first term $E_{1}=\left(\bigoplus_{p=0}^{\infty} E_{1}^{-p, *}, d\right)$ of the cohomology Sinha spectral sequence is the normalized complex Tot $A_{\bullet}=\left(\bigoplus_{p=0}^{\infty} s^{-p} N A_{p}, d\right)$, where $s^{-p}$ denotes $p$-fold desuspension. The differential is, as usual, the alternated sum of faces $d_{i}$.

The Lie algebra $L^{n}, n \geq 0$, is generated by $\alpha_{i j}, 1 \leq i \neq j \leq n$, of degree $d-1$ [16]. The relations are

$$
\begin{array}{ll} 
& \alpha_{j i}=(-1)^{d} \alpha_{i j}, \\
\text { quadratic } & {\left[\alpha_{i j}, \alpha_{k l}\right]=0, \quad \text { if } \#\{i, j, k, l\}=4,} \\
\text { relations } & {\left[\alpha_{i j}, \alpha_{j k}+\alpha_{i k}\right]=0 .}
\end{array}
$$

The bracket in $L^{n}$ is the Whitehead bracket which is of degree -1 .

It is well known that $A_{n}$ and $L^{n}$ are Koszul dual 8 , 5 This means that the $\left(\begin{array}{l}n \\ 2\end{array}\right)$ dimensional space $V_{n}$ of generators of $A_{n}$ is dual to the space $V^{n}$ of generators of $L^{n}$. Also the space $R_{n} \subset S^{2} V_{n}$ spanned by the quadratic relations (3.1) is orthogonal to the space $R^{n} \subset S^{2} V^{n}$ of quadratic relations of $L^{n}$. Moreover $A_{n}$ and $L^{n}$ are Koszul which means some nice homological property of their bar-constructions [25]. This property will be used in the proof of our main result Theorem 3.1.

The cofaces $d^{k}: L^{n} \rightarrow L^{n+1}$, for $k=0, \ldots, n+1$, and the codegeneracies $s^{k}: L^{n} \rightarrow L^{n-1}$, for $k=1, \ldots, n$, are defined on generators as follows:

$$
d^{k}\left(\alpha_{i j}\right)=\left\{\begin{array}{ll}
\alpha_{i j}, & \text { if } i<j<k ; \\
\alpha_{i j}+\alpha_{i, j+1}, & \text { if } i<j=k ; \\
\alpha_{i, j+1}, & \text { if } i<k<j ; \\
\alpha_{i, j+1}+\alpha_{i+1, j+1}, & \text { if } i=k<j ; \\
\alpha_{i+1, j+1}, & \text { if } k<i<j .
\end{array} \quad s^{k}\left(\alpha_{i j}\right)= \begin{cases}\alpha_{i j}, & \text { if } i<j<k ; \\
0, & \text { if } i<j=k ; \\
\alpha_{i, j-1}, & \text { if } i<k<j ; \\
0, & \text { if } i=k<j ; \\
\alpha_{i-1, j-1}, & \text { if } k<i<j .\end{cases}\right.
$$

In particular $d^{0}\left(\alpha_{i j}\right)=\alpha_{i+1, j+1}, d^{n+1}\left(\alpha_{i j}\right)=\alpha_{i j}$.

The normalized part

$$
N L^{n}=\bigcap_{i=1}^{n} \operatorname{ker} s^{i} \subset L^{n}
$$

is spanned by the iterated brackets of generators that use each index $i=1, \ldots, n$. The space $N L^{n}$ is isomorphic to a subspace of a graded free Lie algebra generated by $x_{1}=\alpha_{12}, x_{2}=\alpha_{13}, \ldots, x_{n-1}=\alpha_{1, n-1}$ spanned by the iterated brackets using each $x_{i}, 1 \leq i \leq n-1$.

The first term $\mathcal{E}^{1}=\left(\bigoplus_{p=0}^{\infty} \mathcal{E}_{-p, *}^{1}, d\right)$ of the homotopy Sinha spectral sequence is the normalized complex Tot $L^{\bullet}=\left(\bigoplus_{p=0}^{\infty} s^{-p} N L^{p}, d\right)$.

Here is our main result.

Theorem 3.1. (i) The $\mathcal{E}^{2}$-term of the homotopy Sinha spectral sequence (for $\overline{E m b}$ ) is rationally isomorphic to the primitive part of the homology $E^{2}$-term.

(ii) The $\mathcal{E}_{2}$-term of the cohomotopy Sinha spectral sequence (for $\overline{E m b}$ ) is rationally isomorphic to the primitive part of the cohomology $E_{2}$-term.

\footnotetext{
${ }^{5}$ To be precise the desuspension $s^{-1} L^{n}$ (being the Lie algebra with bracket of degree zero) is the Lie Koszul dual of $A_{n}$. But for technical reasons in the context of the paper it is more convenient to work with $L^{n}$ rather than with $s^{-1} L^{n}$.
} 
Since the $E^{2}$-homology term is a graded polynomial bialgebra 6 assertions (i) and (ii) are equivalent. So, we will prove only (ii). The proof will be given in Section 6 .

\section{Correspondence of Bigradings}

In this section we describe how the homotopy spectral sequence bigradings $\left(p^{\prime}, q^{\prime}\right)$ are related to the homology spectral sequence bigradings $(p, q)$ via the isomorphism of Theorem 3.1.

We will give an heuristic explanation of this correspondence. But one can easily establish it by a simple analysis of the proof given in Section 6 .

A monomial of degree $i$ in $N A_{j}$ is an element of $E_{1}^{p, q}$ with $p=-j, q=(d-1) i$. A Lie monomial of degree $i^{\prime}$ in $N L^{j^{\prime}}$ is an element of $\mathcal{E}_{p^{\prime}, q^{\prime}}^{1}$ with $p^{\prime}=-j^{\prime}, q^{\prime}=$ $(d-1) i^{\prime}-\left(i^{\prime}-1\right)=(d-2) i^{\prime}+1$ (recall that the bracket in $L^{\bullet}$ is of degree -1$)$.

The degrees $i, i^{\prime}$ in both cases will be called complexity. The complexity is preserved by the differential.

Up to a shift of grading the complexes Tot $A \bullet$, Tot $L^{\bullet}$ depend only on the parity of $d$. So it is natural to expect that the isomorphism of Theorem 3.1 respects this periodicity and therefore preserves the complexity. The total grading $p+q, p^{\prime}+q^{\prime}$ must also be unchanged.

Let us find the bigrading $\left(i^{\prime}, j^{\prime}\right)=\left(i, j^{\prime}\right)$ of $\operatorname{Tot} L^{\bullet}$ that should correspond to the bigrading $(i, j)$ of $\operatorname{Tot} A_{\bullet}$. We have

$$
\begin{aligned}
p+q & =(d-1) i-j, \\
p^{\prime}+q^{\prime} & =(d-2) i+1-j^{\prime} .
\end{aligned}
$$

So $p+q=p^{\prime}+q^{\prime}$ implies

$$
j^{\prime}=j-i+1 .
$$

For example if $j=2 i$ (the case of the lower line in $E_{2}$, which corresponds to the bialgebra of chord diagrams), one has $j^{\prime}=i+1$. This corresponds to the lower line in $\mathcal{E}_{2}$.

The case $j=i+1$ (upper line in $E_{2}$ ) produces $j^{\prime}=2$ (right line in $\mathcal{E}_{2}$ ). This situation produces exactly the homotopy and the homology of the factor $\Omega^{2} S^{d-1}$ of $\overline{E m b}$ 32.

In general for a non-trivial monomial of degree $i$ in $N A_{j}$ the number $j-i$ is the number of connected components in the corresponding forest; see Section 3 , So the correspondence (4.1) can be resumed as follows: the number of connected components of forests in the cohomological case corresponds to the number of points (of configuration spaces) minus 1 in the homotopy case.

\section{Fixing NOTATiON}

In this section we review some necessary background and fix some notation.

5.1. $B / B^{2}$. By CDGA we understand the category of graded connected differential graded algebras with differential raising the degree by 1 . Almost all algebras we deal with are 1-connected, i.e. their 1-degree component is trivial.

\footnotetext{
${ }^{6}$ This is true for any field of coefficients 33, Corollary 13.4].
} 
Consider a functor from CDGA to the category of differential graded vector spaces (complexes):

$$
\begin{aligned}
P: \quad \text { CDGA } & \longrightarrow d g-\text { Vect } \\
B & \longmapsto B_{>0} /\left(B_{>0}\right)^{2} .
\end{aligned}
$$

For simplicity of notation $P(B)=B_{>0} /\left(B_{>0}\right)^{2}$ will be denoted by $B / B^{2}$.

5.2. $\mathcal{L}(B)$. By $\mathcal{L}$ we denote the cobar construction

$$
\mathcal{L}: \text { CDGA } \rightarrow d g-\text { coLie },
$$

which assigns to any commutative $d g$-algebra $B$ a free $d g$-Lie coalgebra with cobracket of degree 1 :

$$
\mathcal{L}(B)=\bigoplus_{n \geq 1}\left(\operatorname{coLie}(n) \otimes\left(B_{>0}\right)^{\otimes n}\right)_{S_{n}},
$$

whose differential is a sum of two things - one arising from the initial differential of $B$, the other from multiplication in $B$. A nice explicit description of this construction is given in [30]. Notice that in our construction the degree of each space $\operatorname{coLie}(n)$ is $1-n$.

One has a natural transformation

$$
\begin{array}{cccc}
\alpha: & \mathcal{L} & \longrightarrow & P \\
\mathcal{L}(B) & \stackrel{\alpha_{B}}{\longrightarrow} & B / B^{2}
\end{array}
$$

which is a morphism of complexes sending $\bigoplus_{n>2}\left(\operatorname{coLie}(n) \otimes\left(B_{>0}\right)^{\otimes n}\right)_{S_{n}}$ to zero and coLie(1) $\otimes B_{>0}=B_{>0}$ to the quotient $B_{>0} /\left(B_{>0}\right)^{2}$.

The following is a standard result in rational homotopy theory [13].

Proposition 5.1. If $B$ is free as a graded commutative algebra, then the map $\mathcal{L}(B) \stackrel{\alpha}{\longrightarrow} B / B^{2}$ is a quasi-isomorphism.

5.3. Totalization. Let $V_{\bullet}$ be a simplicial $d g$-vector space. If not stated otherwise, we always assume that the differential raises the degree by 1 . We define Tot $V_{\bullet}$ as a complex whose space is $\bigoplus_{n>0} s^{-n} N V_{n}$ and the differential is the sum of the inner differential of each $V_{n}$ plus the alternated sum of faces. Notice that Tot $V_{\bullet}$ might be negatively graded, however in all the considered cases the totalization always produces positively graded complexes.

Let $V_{\bullet}$ and $W_{\bullet}$ be two simplicial $d g$-vector spaces. Assume that Tot $V_{\bullet}$ and Tot $W$ • are positively graded. One has the Eilenberg-Mac Lane quasi-isomorphism [23, §29]:

$$
\operatorname{Tot} V_{\bullet} \otimes \operatorname{Tot} W_{\bullet} \stackrel{E M}{\longrightarrow} \operatorname{Tot}\left(V_{\bullet} \otimes W_{\bullet}\right) .
$$

This map permits us to define a product on the totalization of any simplicial commutative $d g$-algebra $B_{\bullet}$ :

$$
\operatorname{Tot} B_{\bullet} \otimes \operatorname{Tot} B_{\bullet} \stackrel{E M}{\longrightarrow} \operatorname{Tot}\left(B_{\bullet} \otimes B_{\bullet}\right) \stackrel{\mu_{\bullet}}{\longrightarrow} \operatorname{Tot} B_{\bullet} .
$$

The (iterated) Eilenberg-Mac Lane map has nice properties. It is associative and $S_{n}$-equivariant. This implies that Tot $B$. is a commutative $d g$-algebra. For example, for the simplicial algebra $A_{\bullet}=H^{*}\left(C^{\bullet}\right)$, the product on $\mathcal{A}=\operatorname{Tot} A \bullet$ is a shuffle of diagrams (see Figure 5). 


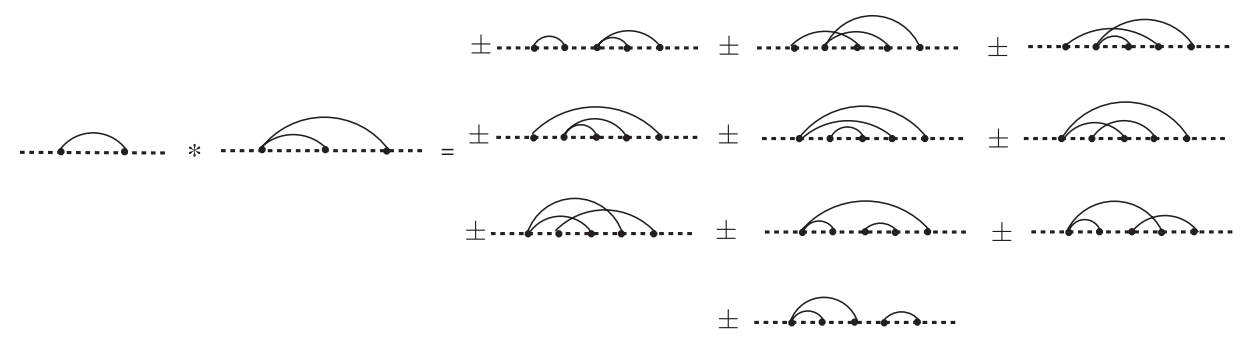

Figure 5. The product in $\mathcal{A}$.

Consider any polynomial functor

$$
\begin{aligned}
\mathcal{P}: \quad d g-\text { Vect } & \longrightarrow d g-\text { Vect } \\
V & \longmapsto \bigoplus_{n \geq 0}\left(\mathcal{P}(n) \otimes V^{\otimes n}\right)_{S_{n}} .
\end{aligned}
$$

The property that the Eilenberg-Mac Lane map is associative and $S_{n}$-equivariant permits us to define a morphism:

$$
\mathcal{P}\left(\operatorname{Tot} V_{\bullet}\right) \stackrel{E M_{\mathcal{P}}}{\longrightarrow} \operatorname{Tot} \mathcal{P}\left(V_{\bullet}\right) .
$$

Lemma 5.2. For a field of characteristic zero, the morphism (5.1) is always a quasi-isomorphism for any simplicial dg-vector space $V_{\bullet}$ (provided Tot $V_{\bullet}$ is positively graded).

Proof. One has that

$$
\left(\operatorname{Tot} V_{\bullet}\right)^{\otimes n} \stackrel{E M_{n}}{\longrightarrow} \operatorname{Tot}\left(V_{\bullet}^{\otimes n}\right)
$$

is an $S_{n}$-equivariant quasi-isomorphism. In characteristic zero it implies that

$$
\left(\mathcal{P}(n) \otimes\left(\operatorname{Tot} V_{\bullet}\right)^{\otimes n}\right)_{S_{n}} \stackrel{E M_{\mathcal{P}(n)}}{\longrightarrow} \operatorname{Tot}\left(\left(\mathcal{P}(n) \otimes V_{\bullet}^{\otimes n}\right)_{S_{n}}\right)
$$

is also a quasi-isomorphism.

Lemma 5.3. For any simplicial commutative dg-algebra $B_{\bullet}$ (provided Tot $B_{\bullet}$ is positively graded) the map $E M_{\mathcal{L}}$ is a quasi-isomorphism:

$$
\mathcal{L}\left(\operatorname{Tot} B_{\bullet}\right) \underset{E M_{\mathcal{L}}}{\stackrel{\simeq}{\longrightarrow}} \operatorname{Tot} \mathcal{L}\left(B_{\bullet}\right) .
$$

Proof. One first has to check that $E M_{\mathcal{L}}$ is a morphism of complexes. This is so because the product part of the differential in $\mathcal{L}\left(\operatorname{Tot} B_{\bullet}\right)$ goes exactly to the product part of the differential in $\operatorname{Tot} \mathcal{L}\left(B_{\bullet}\right)$ (here one uses the fact that the product in Tot $B$. was defined through the Eilenberg-Mac Lane map). To see that $E M_{\mathcal{L}}$ is an isomorphism in homology one can consider the spectral sequences for both complexes assigned to the filtration by the degree $n$ of the polynomial functor $\mathcal{L}=$ $\bigoplus_{n \geq 1} \mathcal{L}_{n}$. It follows from Lemma 5.2 that the induced map of spectral sequences is an isomorphism starting from the first page.

\section{Proof of Theorem 3.1}

The proof is the following sequence of quasi-isomorphisms:

$$
\mathcal{A} / \mathcal{A}^{2} \underset{\alpha}{\stackrel{\simeq}{\simeq}} \mathcal{L}(\mathcal{A})=\mathcal{L}\left(\operatorname{Tot} A_{\bullet}\right) \underset{E M_{\mathcal{L}}}{\simeq} \operatorname{Tot} \mathcal{L}\left(A_{\bullet}\right) \stackrel{\simeq}{\longleftarrow} \operatorname{Tot}\left(L_{\bullet}\right) .
$$


It is well known that $\mathcal{A}=\operatorname{Tot} A \bullet$ is a commutative non-cocommutative $d g$-bialgebra, moreover its homology bialgebra $H^{*}(\mathcal{A})$ is graded polynomial [31, 32. We have by Proposition 5.1 that the first arrow $\alpha$ is a quasi-isomorphism. So the homology of both complexes $\mathcal{A} / \mathcal{A}^{2}, \mathcal{L}(\mathcal{A})$ is the space of generators of $H^{*}(\mathcal{A})$ which is exactly the space of primitives.

The second arrow is a quasi-isomorphism by Lemma 5.3 .

Let us explain the last quasi-isomorphism

$$
\operatorname{Tot}\left(L_{\bullet}\right) \stackrel{\simeq}{\longrightarrow} \operatorname{Tot} \mathcal{L}\left(A_{\bullet}\right) .
$$

The algebra $L^{n}$ is the Lie Koszul dual of $A_{n}$ [8]. One has the natural inclusion (of Lie coalgebras):

$$
L_{n} \hookrightarrow \mathcal{L}\left(A_{n}\right) .
$$

The map (6.3) describes the so called "diagonal" homology of $\mathcal{L}\left(A_{n}\right)$. The property $A_{n}$ is Koszul means that $\mathcal{L}\left(A_{n}\right)$ has only diagonal (non-trivial) homology. In other words (6.3) is a quasi-isomorphism. But (6.3) is a simplicial morphism. As a consequence (6.2) is also a quasi-isomorphism.

To see that the bigradings correspond by the way described in Section 4 , one should generalize the grading complexity on all the intermediate complexes of the zig-zag (6.1), and to show that all the morphisms preserve it. We leave it as an exercise to the reader.

\section{Part 2. Graph-complexes}

\section{INTRODUCTION}

Graph-complexes are widely used to study the homology of interesting spaces and to prove interesting theorems [10, 11, 12, 14, 15, 17, 18, 20, 24. One series of such graph-complexes (its slight modification will be denoted by $\left\{D_{n}\right\}_{n \geq 0}$ throughout the paper) was used by M. Kontsevich to prove the formality of the operad of little $d$-cubes [19. The idea of the proof is that $\left\{D_{n}\right\}_{n \geq 0}$ are quasi-isomorphic to the cochains of the operad, and one also has projections inducing the homology isomorphism

$$
D_{n} \underset{\bar{I}_{n}}{\stackrel{\simeq}{\longrightarrow}} A_{n}=H^{*}(C\langle n\rangle) .
$$

$A_{n}$ is considered as a commutative $d g$-algebra with zero differential.

A more thorough account on this result was given by I. Volić and the first author in 22 .

In [5, 6] another graph-complex was defined (its slight modification will be denoted by $\mathcal{D}$ in the paper). By means of integration over configuration spaces this complex was naturally mapped to the De Rham complex of the space Emb of long knots. One conjectures that this map is a quasi-isomorphism. The reason why it might be so is that $\mathcal{D}$ is quasi-isomorphic to $\mathcal{A}=\operatorname{Tot} A$. (see Theorem 8.6), and therefore the homology of $\mathcal{D}$ is exactly the rational homology of $E m b$.

The complexes $\left\{D_{n}\right\}_{n \geq 0}$ form a simplicial commutative $d g$-algebra. Its totalization Tot $D_{\bullet}$ is exactly the complex $\mathcal{D}$. 
Another motivation for us to study graph-complexes is that they generalize on higher dimensional homology of knot spaces the 3-valent diagrams calculus developed by Dr. Bar Natan [4] (in the relation with the finite type knot invariants). For example, Theorem 6 of [4, which says that the bialgebra of chord diagrams is isomorphic to the bialgebra of 3 -valent diagrams, is an obvious consequence of the fact that $\mathcal{A}$ is quasi-isomorphic to $\mathcal{D}$ : the lower line homology of the dual to $\mathcal{A}$ is the bialgebra of chord diagrams and the lower line homology of the dual to $\mathcal{D}$ is the bialgebra of 3 -valent diagrams modulo $S T U, A S$, and $I H X$ relations.

In Section 9 we define a new series of graph-complexes $\left\{P_{n}\right\}_{n \geq 0}$ satisfying $H^{*}\left(P_{n}\right)$ $=L_{n}=\operatorname{Hom}\left(\pi_{*}\left(C^{n}\right), \mathbb{Q}\right)$. We show that the totalization complex $\mathcal{P}=$ Tot $P \bullet$ is quasi-isomorphic to Tot $L_{\bullet}$ and therefore $H^{*}(\mathcal{P})=H o m\left(\pi_{*}(\overline{E m b}), \mathbb{Q}\right)$.

\section{Cohomology GRAPH-COMPLEX FOR CONFIGURATION AND KNOT SPACES}

Our definition of the space $D_{n}$ of diagrams is very close to that of [19, 22].

A diagram $\Gamma$ on $n$ external and $q$ internal vertices is any graph with $n$ external vertices (lying on the line $\mathbb{R}^{1}$ and labeled consequently $1,2, \ldots, n$ ) and $q$ (nonlabeled) internal vertices, and some number of oriented segments connecting them. Those segments that connect two external vertices are called chords and all others are edges.

The orientation set of a diagram is the union of the set of internal vertices (such elements are considered to be of degree $-d$ ) and the set of chords and edges (such elements are of degree $d-1$ ). An orientation of a diagram is any ordering of its orientation set. The degree of a diagram is the total degree of the elements from the orientation set.

Definition 8.1. A diagram is called admissible if

(1) it does not contain an internal vertex of valence $\leq 2$;

(2) it contains neither edges nor chords connecting a vertex to itself (no loops);

(3) every internal vertex is connected by a path to an external one.

Remark 8.2. The distinction between our definition and the one given in [19, 22] is that we do permit multiple edges and multiple chords. This will be important for Theorem 9.3. This difference is essential only if $d$ is odd, for even $d$ graphs with multiple edges/chords cancel out by the orientation relation; see below.

Definition 8.3. The space $D_{n}$ is defined as the $\mathbb{Q}$-vector space spanned by the admissible diagrams $\Gamma$ with $n$ external vertices, modulo the relations

(1) if $\Gamma_{1}$ and $\Gamma_{2}$ differ only by an orientation of an edge, then

$$
\Gamma_{1}=(-1)^{d} \Gamma_{2}
$$

(2) if $\Gamma_{1}$ and $\Gamma_{2}$ differ only by a permutation of the orientation set, then

$$
\Gamma_{1}= \pm \Gamma_{2}
$$

where the sign is the Koszul sign of permutation (taking into account the degrees of elements). 
$D_{0}$ is defined to be $\mathbb{Q}$ being spanned by the empty diagram.

The differential in $D_{n}$ is defined as the sum of contractions of edges.

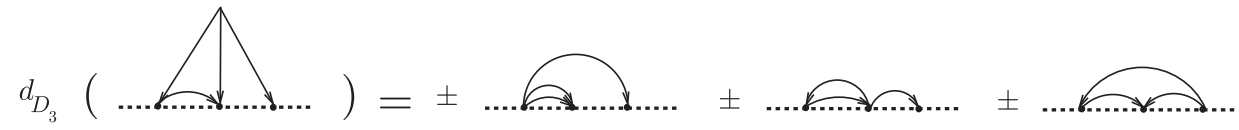

Figure 6. The differential in $D_{3}$.

For the signs convention, see [19, 22].

The multiplication in $D_{n}$ is defined by superimposing:

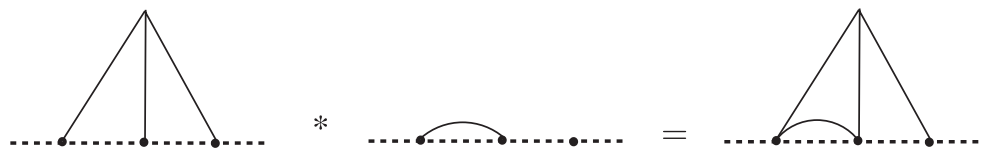

Figure 7 . The product in $D_{3}$.

Proposition $8.4([19,22])$. The complexes $D_{n}, n \geq 0$, with multiplication as above are commutative dg-algebras.

Lemma-definition 8.5. The morphisms

$$
\bar{I}_{n}: D_{n} \rightarrow A_{n},
$$

which send any diagram having internal vertices to zero, and all others to the corresponding monomials in $A_{n}$ (see Section 3), are quasi-isomorphisms of commutative dg-algebras.

Proof. Our complexes are slightly different from those used in [19, 22], but the proof is the same; see 22.

The complexes $\left\{D_{n}\right\}_{n \geq 0}$ form a cooperad in CDGA. For the definition of structure maps see [19, 22]. This cooperad is endowed with a morphism to the cooperad $\mathcal{C O} \mathcal{A S S}$ :

$$
D_{\bullet} \rightarrow \mathcal{C O} \mathcal{A S S} \text {. }
$$

Any non-trivial diagram is sent to zero and the trivial diagram with $n$ external vertices is sent to $1 \in \mathbb{Q}=\mathcal{C O} \mathcal{A S S}(n)$. This endows $D$. with a structure of a simplicial commutative $d g$-algebra; see Section 2, The simplicial structure of $D$. is completely analogous to that of $A_{\bullet}$; see Figures 3 and 4 . For $\Gamma \in D_{n}$, the face map $d_{0}$ removes the vertex 1 if it was of valence 0 , otherwise $d_{0}(\Gamma)=0$. The face $d_{i}(\Gamma), i=1, \ldots, n-1$, is obtained by contracting the segment $[i, i+1]$ of $\mathbb{R}^{1}$. Finally $d_{n}$ removes the last point $n$ (if it was of valence 0 , otherwise $d_{n}(\Gamma)=0$ ). The degeneracy $s_{i}, i=1, \ldots, n+1$, is defined as insertion of a new external point between $i-1$ and $i$.

The normalized part $N D$ • of $D$ • is spanned by the diagrams whose all external vertices are of positive valence. We will define a graph-complex $\mathcal{D}$ as the totalization of $D$. 
Theorem 8.6. The complex $\mathcal{D}=\operatorname{Tot} D$. is quasi-isomorphic to $\mathcal{A}=\operatorname{Tot} A$. and therefore the homology of $\mathcal{D}$ is the rational cohomology of $\overline{E m b}$ :

$$
H^{*}(\mathcal{D})=H^{*}(\overline{E m b}) \text {. }
$$

Proof. The map (8.1) is a quasi-isomorphism of simplicial commutative $d g$-algebras, which induces a quasi-isomorphism of totalizations:

$$
\mathcal{D}=\operatorname{Tot}\left(D_{\bullet}\right) \stackrel{\simeq}{\longrightarrow} \operatorname{Tot}\left(A_{\bullet}\right)=\mathcal{A}
$$

But $H^{*}(\mathcal{A})=E_{2}^{*, *}\left(C^{\bullet}\right)=H^{*}(\overline{E m b})$. (We use the fact that the homology Sinha spectral sequence collapses rationally at $E^{2}$ [21.)

Since $d_{0}$ and $d_{n}$ always act as zero on $N D_{n}$, the differential in $\mathcal{D}$ is the sum of contractions of edges and of line segments of $\mathbb{R}^{1}$; see Figure 8 .

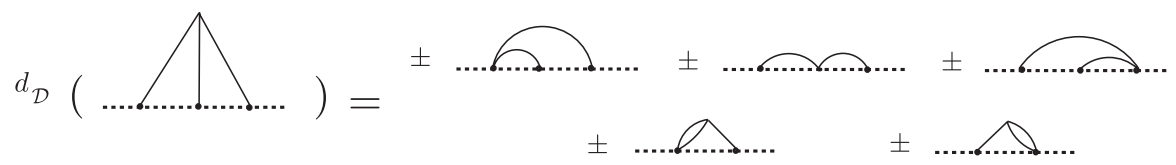

Figure 8. The differential in $\mathcal{D}$.

In $\mathcal{D}=$ Tot $D$. a degree of a graph $\Gamma \in D_{n}$ is desuspended by $n$. Geometrically we add to the orientation set of $\Gamma n$ elements of degree -1 that correspond to the external vertices of $\Gamma$. The product in $\mathcal{D}$, which is defined via the EilenbergMac Lane map, acts as a shuffle of external points. For each summand the ordering of its orientation set is obtained by concatenation.

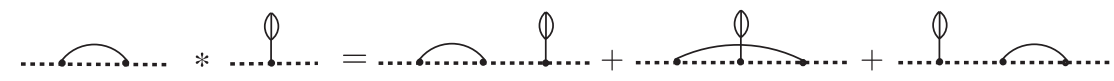

Figure 9. The product in $\mathcal{D}$.

The coproduct in $\mathcal{D}$ is the coconcatenation.

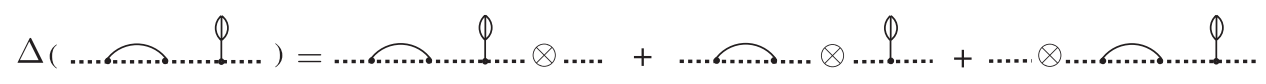

FiguRE 10. The coproduct in $\mathcal{D}$.

\section{COHOMOTOPy GRAPH-COMPleX FOR CONFIGURATION AND KNOT SPACES}

A non-trivial graph $\Gamma \in D_{n}$ is called non-decomposable if it cannot be represented as a product $\Gamma=\Gamma_{1} \cdot \Gamma_{2}$ of two non-trivial graphs $\Gamma_{1}, \Gamma_{2} \in D_{n} 7$ The space spanned by non-decomposable graphs will be denoted by $P_{n}$.

Remark 9.1. In other words, a graph is non-decomposable if it is connected (and non-empty) when we remove from it all the external vertices with their little neighborhoods.

\footnotetext{
${ }^{7}$ We consider the inner product of $D_{n}$; see Figure 7
} 
Notice however that a non-decomposable graph might be disconnected: together with its main connected part it can have a number of singletons - external vertices of valence 0 .

The complex $P_{n}$ is a quotient-complex of $D_{n}$. It is easy to see that $P_{n}=D_{n} / D_{n}^{2}$.

Proposition 9.2. $D_{n}$ is a free graded commutative algebra whose space of generators is $P_{n}$.

Proof. To see this, one can remove the external vertices and consider the resulting connected components. It is here where it is important that we permit multiple edges/chords.

Theorem 9.3. The homology of $P_{n}$ is the rational cohomotopy of the configuration space:

$$
H^{*}\left(P_{n}\right)=L_{n}=\operatorname{Mor}\left(\pi_{*}\left(C^{n}\right), \mathbb{Q}\right) .
$$

Proof. We have the quasi-isomorphisms

$$
P_{n}=D_{n} / D_{n}^{2} \underset{\simeq}{\stackrel{\simeq}{\alpha}} \mathcal{L}\left(D_{n}\right) \stackrel{\simeq}{\longrightarrow} \mathcal{L}\left(A_{n}\right) \stackrel{\simeq}{\longleftarrow} L_{n} .
$$

The first arrow $\alpha$ is a quasi-isomorphism by Proposition 5.1, the second one is induced by the quasi-isomorphism (8.1), the last one is due to the Koszul property.

It follows from Remark 9.1 that $P_{\bullet}$ is a simplicial subspace of $D_{\bullet}$ - the simplicial structure maps preserve $P_{\bullet}$. Its normalized part $N P_{\bullet}$ is spanned by the connected non-decomposable diagrams, i.e. by the diagrams without singletons. Now define a complex $\mathcal{P}$ as the totalization of $P_{\boldsymbol{~}}$. Obviously, $\mathcal{P}$ is a quotient-complex of $\mathcal{D}$.

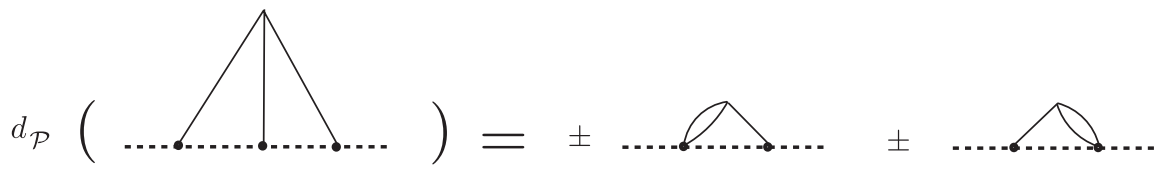

Figure 11. The differential in $\mathcal{P}$.

Theorem 9.4. The complex $\mathcal{P}=\operatorname{Tot} P_{\bullet}$ is quasi-isomorphic to Tot $L \bullet$ and therefore the homology of $\mathcal{P}$ is the rational cohomotopy of $\overline{E m b}$ :

$$
H^{*}(\mathcal{P})=\operatorname{Mor}\left(\pi_{*}(\overline{E m b}), \mathbb{Q}\right) .
$$

Proof. Diagram (9.1) is a sequence of quasi-isomorphisms of simplicial $d g$-spaces. Passing to totalization one gets the result. (Tot $L_{\bullet}$ computes the rational cohomotopy of $\overline{E m b}$ since the homotopy Sinha spectral sequence collapses at $E^{2}$ [2], which is also a consequence of the homology spectral sequence collapse at $E^{2}$ [21, and Theorem 3.1.)

Remark 9.5. $P \bullet$ is a simplicial $L_{\infty}$-coalgebra. Indeed, given a free commutative $d g$-algebra $B$, any section $B / B^{2} \hookrightarrow B$ of the projection $B \rightarrow B / B^{2}$ defines an 
$L_{\infty}$-coalgebra structure on $B / B^{2}$. We have natural inclusions

$$
D_{n} / D_{n}^{2}=P_{n} \hookrightarrow D_{n} .
$$

Since (9.2) is a map of simplicial vector spaces $P_{\bullet} \rightarrow D_{\bullet}$, the $L_{\infty}$-coalgebra operations on $P_{n}, n \geq 0$, commute with the simplicial structure maps.

We finish by giving some examples of cycles in $\mathcal{P}$. Obviously the diagram is the

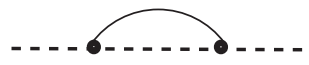

first non-trivial cycle (for both even and odd $d$ ). Its degree is $d-3$. It can be easily seen that the sum of diagrams (taken with appropiate signs)

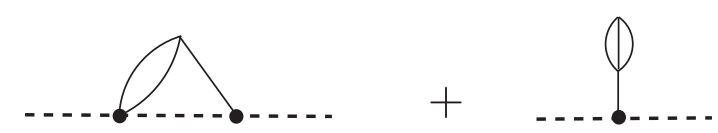

is a non-trivial cycle in the case when $d$ is odd (and therefore multiple edges are possible). The degree of this cycle is $2 d-5$. Recall that $\overline{E m b}$ is homotopy equivalent to $E m b \times \Omega^{2} S^{d-1}$. The above cycles describe the rational cohomotopy coming from the second factor $\Omega^{2} S^{d-1}$ (for which the rational cohomotopy of the sphere $S^{d-1}$ is shifted by 2 ).

The first non-trivial cohomotopy coming from the first factor $E m b$ is of degree $2 d-6[32$. In case of even $d$ it is given by the diagram:

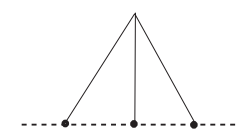

For odd $d$ it is given by the sum:

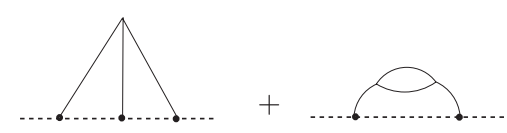

\section{ACKNOWLEDGEMENTS}

The second author thanks D. Sinha, J. Conant, and L. Ionescu for fruitful conversations. We thank R. Budney for an attentive reading and various corrections.

\section{REFERENCES}

[1] V. Arnol'd. The cohomology ring of the group of colored braids. (Russian) Mat. Zametki 5, 1969, pp 227-231. MR0242196 (39:3529)

[2] G. Arone, P. Lambrechts, V. Turchin, I. Volić. Coformality and rational homotopy groups of spaces of long knots. To appear in Math. Res. Letters. Preprint arXiv:math/0701350

[3] G. Arone, P. Lambrechts, I. Volić. Calculus of functors, operad formality, and rational homology of embedding spaces. To appear in Acta Math. Preprint math.AT/0607486. 
[4] Dr. Bar Natan. On the Vassiliev knot invariants. Topology 34 (1995), no. 2, 423-472. MR:1318886 (97d:57004)

[5] A. Cattaneo, P. Cotta-Ramusino, R. Longoni. Configuration spaces and Vassiliev classes in any dimension. Algeb. Geom. Topol., 2: 949-1000, 2002. MR1936977|(2004a:57014)

[6] A. Cattaneo, P. Cotta-Ramusino, R. Longoni. Algebraic structures on graph cohomology. J. Knot Theory Ramifications 14 (2005), no. 5, 627-640. MR2162117 (2006g:58021)

[7] F. Cohen. The homology of $C_{n+1}$ spaces. In Lecture Notes in Mathematics, Vol. 533, 1976.

[8] F. Cohen, S. Gitler. Loop spaces of configuration spaces, braid-like groups, and knots. Cohomological methods in homotopy theory (Bellaterra, 1998), 59-78, Progr. Math., 196, Birkhauser, Basel, 2001. MR1851248 (2003c:55007)

[9] J. Conant. Homotopy approximations to the space of knots, Feynman diagrams, and a conjecture of Scannell and Sinha, math.GT/0601647.

[10] J. Conant, K. Vogtmann. Infinitesimal operations on complexes of graphs. Math. Ann. 327 (2003), no. 3, 545-573. MR2021029 (2004m:17026)

[11] J. Conant, K. Vogtmann. On a theorem of Kontsevich. Algebr. Geom. Topol. 3 (2003), 11671224. MR2026331 (2004m:18006)

[12] M. Culler, K. Vogtmann. Moduli of graphs and automorphisms of free groups. Invent. Math. 84 (1986), no. 1, 91-119. MR830040 (87f:20048)

[13] Y. Felix, S. Halperin, J.-C. Thomas. Rational homotopy theory. Graduate Texts in Mathematics, 205. Springer-Verlag, New York, 2001. xxxiv+535 pp. MR.1802847 (2002d:55014)

[14] D. Fiorenza, L. Ionescu. Graph complexes in deformation quantization. Lett. Math. Phys. 73 (2005), no. 3, 193-208. MR2188293 (2007d:53153)

[15] E. Getzler, M. Kapranov. Modular operads. Compositio Math. 110 (1998), no. 1, 65-126. MR1601666 (99f:18009)

[16] T. Kohno. Loop spaces of configuration spaces and finite type invariants. Invariants of knots and 3-manifolds (Kyoto, 2001), 143-160 (electronic), Geom. Topol. Monogr., 4, Geom. Topol. Publ., Coventry, 2002. MR2002608 (2004g:55014)

[17] M. Kontsevich. Formal (non)commutative symplectic geometry. The Gel'fand Mathematical Seminars, 1990-1992, 173-187, Birkhäuser Boston, Boston, MA, 1993. MR1247289 (94i:58212)

[18] M. Kontsevich. Feynman diagrams and low-dimensional topology. In Volume II of Progress in Mathematics 120, 1994. MR1341841 (96h:57027)

[19] M. Kontsevich. Operads and motives in deformation quantization. Lett. Math. Phys 48(1): 35-72, 1999. MR1718044 (2000j:53119)

[20] M. Kontsevich. Deformation quantization of Poisson manifolds. Lett. Math. Phys. 66 (2003), no. 3, 157-216. MR2062626 (2005i:53122)

[21] P. Lambrechts, V. Turchin, I. Volić. The rational homology of spaces of long knots in codimension $>2$. Preprint math/0703649.

[22] P. Lambrechts, I. Volić. Formality of the little $d$-discs operad. In preparation.

[23] J. P. May. Simplicial objects in algebraic topology. Van Nostrand Mathematical Studies, No. 11 D. Van Nostrand Co., Inc., Princeton, N.J.-Toronto, Ont.-London 1967 vi+161 pp. MR0222892 (36:5942)

[24] R. C. Penner. Perturbative series and the moduli space of Riemann surfaces. J. Differential Geom. 27 (1988), no. 1, 35-53. MR.918455 (89h:32045)

[25] S. Priddy. Koszul resolutions. Trans. Amer. Math. Soc. 152 (1970), 39-60. MR0265437 $(42: 346)$

[26] P. Salvatore. Knots, operads and double loop spaces. Int. Math. Res. Not. 2006, Art. ID 13628, 22 pp. MR2276349 (2007j:57012)

[27] K. Scannell, D. Sinha. A one-dimensional embedding complex. J. Pure Appl. Algebra, 170(1): 93-107, 2002. MR.1896343 (2003b:55012)

[28] D. Sinha. The topology of spaces of knots. math.AT/ 0202287 v6, 2007.

[29] D. Sinha. Operads and knot spaces. J. Amer. Math. Soc. 19 (2006), no. 2, 461-486. MR2188133 (2006k:57070)

[30] D. Sinha, B. Walter. Lie coalgebras and rational homotopy theory I, math.AT/0610437.

[31] V. Turchin (Tourtchine). On the homology of the spaces of long knots. Advances in topological quantum field theory, 23-52, NATO Sci. Ser. II Math. Phys. Chem., 179, Kluwer Acad. Publ., Dordrecht, 2004. MR2147415 (2006d:55011) 
[32] V. Turchin (Tourtchine). On the other side of the bialgebra of chord diagrams. Journal of Knot Theory and its Ramifications. Vol. 16 (5), May 2007, pp. 575-629. MR2333307

[33] V. Turchin (Tourtchine). What is one-term relation for higher homology of long knots. Moscow Mathematical Journal, Vol. 6 (1), 2006, pages 169-194, $223 . \quad$ MR2265954 (2007h:57036)

Institut Mathématique, University Catholique de Louvain, 2 Chemin du Cyclotron, B-1348 Louvain-la-Neuve, Belgium

E-mail address: lambrechts@math.ucl.ac.be

$U R L:$ http://milnor.math.ucl.ac.be/plwiki

Department of Mathematics, University of Oregon, Eugene, Oregon 97403 - and Institut des Hautes Études Scientifiques, 91440 Bures-sur-Yvette, France

Current address: Department of Mathematics, Kansas State University, Manhattan, Kansas 66506

E-mail address: turchin@math.ksu.edu 